\title{
Discrimination of geons by pigeons: The effects of variations in surface depiction
}

\author{
MICHAEL E. YOUNG, JESSIE J. PEISSIG, and EDWARD A. WASSERMAN \\ University of Iowa, Iowa City, Iowa \\ and \\ IRVING BIEDERMAN \\ University of Southern California, Los Angeles, California
}

\begin{abstract}
We explored how changes in the depiction of the surface features of a simple volume (a geon) affected the pigeon's recognition performance. Pigeons were trained to make a different keypeck response to each of four computer-rendered single-geon objects. In Experiment 1, the pigeons were tested with images of the original stimuli in which the light source was shifted from its original position, as well as with silhouettes and line drawings of these objects. All three types of stimulus variations resulted in marked drops in performance: above chance for silhouettes and light-change stimuli, but at chance for line drawings. In Experiment 2, the pigeons were tested with images of the original stimuli in which the contrast levels were either increased or decreased. These transformations resulted in very small drops in performance (except for the complete absence of contrast-a silhouette). These results indicated that the pigeons attended to the shape of the outside contour of an object and to the relative brightness of an object's surface contours.
\end{abstract}

Object recognition is a pervasive and yet surprisingly complex cognitive skill. The visual system must map a variety of different retinal images to the same cognitive concept. This mapping process may need to be very general (as is necessary when you categorize a stimulus as a car), or it may need to be quite specific (as when you recognize your own car among a collection of similar vehicles). Our research on object recognition in the pigeon has focused on examining the pigeon's propensity to place variations of an object into the same category as the originally trained object. ${ }^{1}$ We have previously documented the behavioral impact of changing a simple object's viewpoint (Peissig, Young, Wasserman, \& Biederman, 1999a, 2000) and its size (Peissig, Young, Wasserman, \& Biederman, 1999b, November), thus documenting the degree of rotational and size invariance that is evidenced in an avian species. In the present study, we focus on the role of the internal structure of the object-those cues that are not derivable from the shape of the object's outline.

\section{Prior Research Involving People}

Intuitively, we would expect the internal features of the stimuli (i.e., edges marking orientation and depth discon-

The data from Experiment 1 were presented at the 1999 meeting of the annual workshop on Object Perception and Memory (OPAM 99), Los Angeles. National Institute of Mental Health Grant MH 473131 supported this research. We thank Christina Antes, Michael Prier, and Diane Scott for their assistance in conducting the experiments and in data analysis. Correspondence concerning this article should be addressed to M. E. Young, now at the Department of Psychology, Southern Illinois University, Carbondale, IL 62901, or to J. J. Peissig, Department of Psychology, University of Iowa, Iowa City, IA 52242-1407 (e-mail: meyoung@siu.edu or jessie-peissig@uiowa.edu). tinuities and shading) to play an important role in recognition. However, good recognition is possible after viewing only the silhouette of an object. In a study by Hayward (1998), human subjects exhibited significant recognition priming when the prime was the silhouette (the filled bounded contour) of an object. More important, this priming effect was only slightly smaller than that observed with a fully textured rendition of the object. Similarly, Newell and Findlay (1997, Experiment 4) found that significantly more errors were made to silhouettes of objects than to their shaded equivalents, but this difference was only revealed for unconventional views of the tested objects (e.g., a top-down view of a bottle or a side view of a frying pan). There were no reliable differences in error rates or response times for the more conventional object views. This set of results indicates that the silhouette of an object is sufficient for successful classification, as long as it clearly depicts the object's parts (Biederman, 1988).

Although an object's silhouette may be sufficient for good object recognition, Biederman and Ju (1988) reported that a line drawing of an object is also sufficient for good recognition by people. A line drawing represents only the object's orientation and depth discontinuities, with color, luminance variations, and texture removed. Biederman and Ju contrasted object recognition performance (as measured by error rates and response times) for color photographs and for line drawings; they reported minimal differences in recognition performance for the two types of stimuli. The studies by Biederman and Ju, Hayward (1998), and Newell and Findlay (1997) suggest that shading, color, and texture are of minimal importance for many object recognition tasks. Biederman and $\mathrm{Ju}$ do note, however, that color and texture play 
a larger role when one is discriminating objects that cannot be distinguished by their shape (e.g., distinguishing a lime from a lemon or identifying your favorite shirt in a pile of laundry).

Although we have suggested that the internal contours and surface specifications of an object are not always necessary for object recognition, we do not mean to imply that they play no role at all. In addition to the experiments by Hayward (1998) and Newell and Findlay (1997) that revealed small effects of removing internal contours and features, a study by Tarr, Kersten, and Bülthoff (1998) suggested that changes in object lighting can have a small but measurable effect on object recognition performance. Changing the direction of object illumination alters the luminance variation of an object, whereas the outline shape of the object and its internal image edges remain unchanged. In a sequential matching task, Tarr et al. (1998) reported that a change in lighting direction produced a significant increase in reaction times in their human observers; but consistent with the other studies that we have discussed, the observed performance differences were relatively small (approximately $20 \mathrm{msec}$ ). It is not clear whether this result represented an effect of light source change per se or just an effect from any display change, as would be produced, for example, by slightly shifting the position of the object (Biederman \& Bar, 1999).

In general, changes in the direction of lighting and the removal of internal surface specifications (such as texture, color, or luminance gradients) produce, at best, only modest effects on human recognition performance for shapedistinctive stimuli. The same, however, may not be true in other species.

\section{Prior Research Involving Pigeons}

Although there are many studies reporting considerable convergence between human and pigeon performance across object recognition tasks (e.g., Bhatt \& Wasserman, 1989; Kirkpatrick-Steger \& Wasserman, 1996; Wasserman et al., 1996; Wasserman, KirkpatrickSteger, Van Hamme, \& Biederman, 1993), there are also studies documenting important differences between the species. For example, the performance of pigeons is more disrupted by the presence of an occluder (Sekuler, Lee, $\&$ Shettleworth, 1996), shows poorer generalization to line drawings (see Biederman \& Ju, 1988; Cabe, 1976), and shows less generalization to depth rotations of a trained stimulus (Peissig et al., 1999a, 2000). Given these differences, we wished to determine whether the sufficiency of silhouettes and line drawings in people, as well as the small effects of illumination changes, would also be found in pigeons. Documenting the relative importance of these cues is critical to the development of an accurate theory of avian object recognition.

Early work on pigeon object recognition has provided evidence that pigeons can recognize objects from silhouettes, but not from line drawings (Cabe, 1976; Cook, Wright, \& Kendrick, 1990; Delius, 1992; Lumsden, 1977).
Careful scrutiny of these studies, however, indicates potential limitations in the generality of their results.

For example, Cook et al. (1990) trained pigeons to discriminate between black-and-white shaded line drawings of birds and mammals. These shaded line drawings were cartoon-like representations of photographs. Cook et al. examined the pigeons' discrimination when given the outline of the figure (eliminating all lines and shading within the body of the figure) and when given the silhouette of the figure (produced by blackening in the outline figure). Their pigeons failed to produce above-chance discrimination to the outline of the figure, but they did produce above-chance discrimination of the figure silhouettes. Despite their above-chance performance, however, the pigeons showed a large drop in accuracy for the silhouettes. Although Cook et al. had good reasons for using blackand-white drawings, these drawings lack the subtle lighting cues that are present in photographs and in computerrendered volumes (e.g., Cook \& Katz, 1999; Peissig et al., 2000; Tarr, Bülthoff, Zabinski, \& Blanz, 1997). The absence of these cues potentially limits the conclusions that can be drawn from this study.

In contrast to Cook et al.'s (1990) use of shaded line drawings during training, Cabe (1976) and Lumsden (1977) trained pigeons to discriminate real, three-dimensional objects that were viewed through a hole in one end of an experimental chamber. Cabe trained pigeons to discriminate between white objects (a cross and a rectangular block, both against a navy gray background) and examined transfer performance to black-and-white photographs, perspective line drawings, and white-on-black silhouettes. He found that black-and-white photographs and white-on-black silhouettes produced performance that was equivalent to that observed to the original objects. (Performance was assessed in a negative transfer task in which the pigeons were required to reverse their previously learned discrimination.) Line drawings, however, were not treated as equivalent.

Cabe's (1976) task did not permit an inference of whether the line drawings produced above-chance transfer. Nevertheless, Cabe's study does provide good evidence that pigeons that are trained to discriminate between threedimensional objects will transfer their discrimination to photographs and silhouettes, but not to line drawings. These findings do not, however, tell us whether pigeons that are trained with two-dimensional images will generalize their performance to silhouettes or to line drawings of those images.

In a similar although very limited study, Lumsden (1977) trained a single pigeon on a go/no-go task to discriminate one view of a white geometric object from a range of views of two similar objects. The pigeon was subsequently tested with a photograph of the original object and with a line drawing/silhouette of the object. The line drawing/silhouette was a line drawing with a black background and a white interior; it thus had the features of a silhouette and a line drawing. During testing, the photo- 

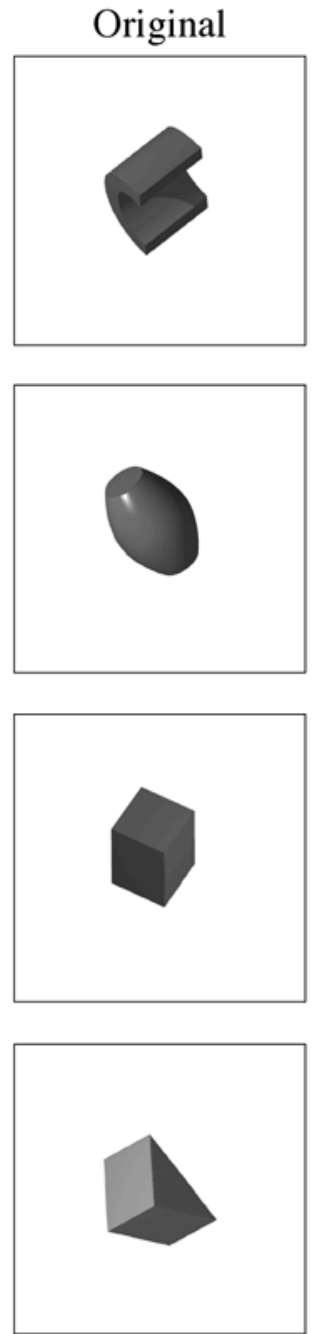

Figure 1. The original and transformed versions of the $0^{\circ}$ The original views (left column) are those used in training.

graph was functionally equivalent to the original object, but the line drawing/silhouette produced a large decrement in response rate that was approximately half of that observed to the original object and the photograph. Responses to the drawing/silhouette, however, were faster to the view to which the pigeon was trained than to the other views, thus providing evidence of above-baseline transfer from the object to the drawing/silhouette.

In contrast to these studies indicating good, although perhaps incomplete, transfer to silhouettes and poor transfer to line drawings, Lombardi and Delius (1988) found excellent transfer for both types of transformations. Their pigeons, however, were trained to discriminate simple geometric shapes that lacked any surface variation. Thus, it was not surprising that the pigeons would show strong transfer, because no information was lost when the pigeons were presented with a silhouette or a line drawing-

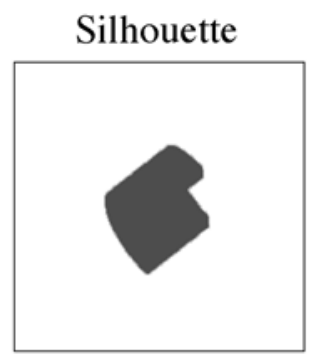

Line Drawing
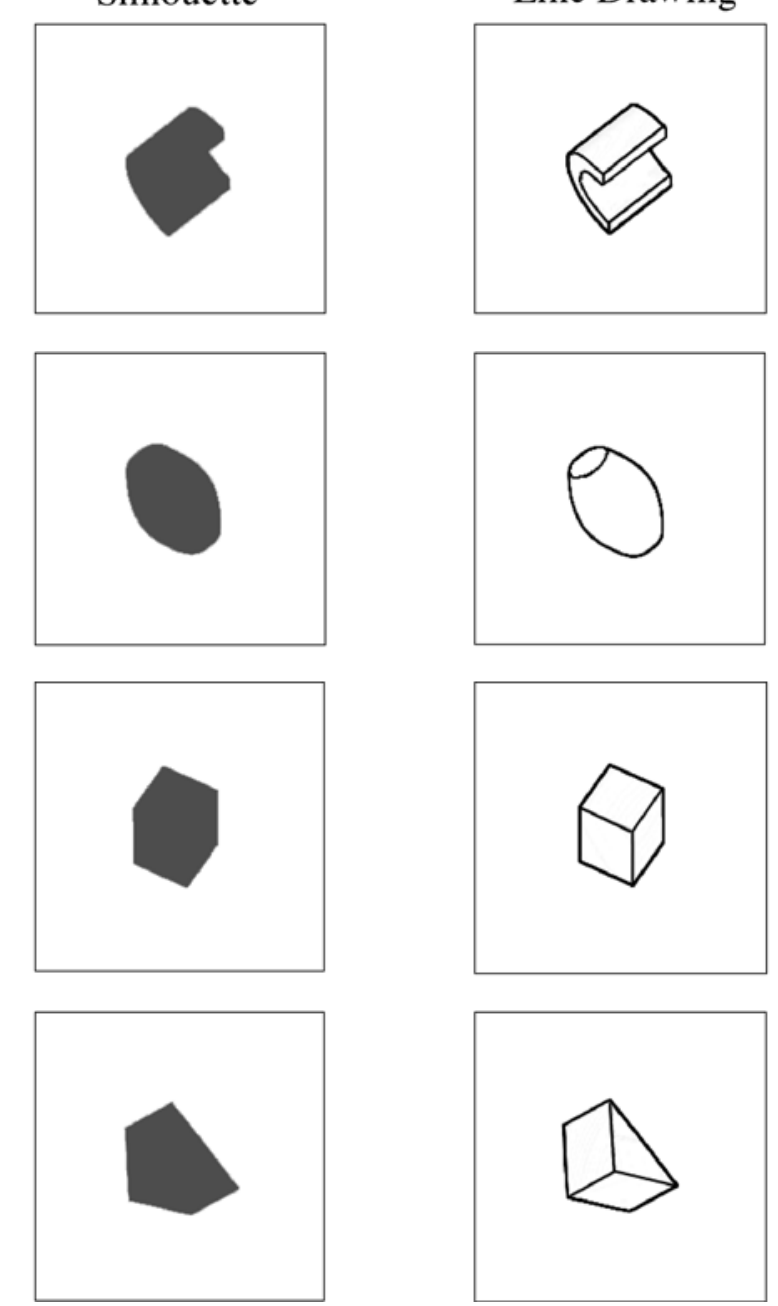

the outline was their only basis of discrimination during training.

\section{The Role of Internal Surface Features}

Our intention was to examine the extent to which pigeons rely on internal surface variations and internal contours when discriminating realistically illuminated, grayscale, two-dimensional renderings of three-dimensional volumes. The pigeons were initially trained with images of a single view of each of four single geons (simple volumetric objects; see Biederman, 1987) in a four-alternative forced-choice task (as is shown in the leftmost column of Figure 1). In Experiment 1, the birds were later tested for their ability to recognize the same objects (1) under a change in the direction of illumination, (2) with all of the internal shading information averaged to produce a silhouette, and (3) with all of the internal shading information 
removed, thus producing a line drawing of the original object (as is shown in the three right-most columns of Figure 1).

Because we found large performance decrements after all of the image transformations used in Experiment 1, in Experiment 2, we examined stimulus transformations that preserved relative shading but still involved significant alterations to the image. New birds were tested for their ability to recognize the same objects after a series of transformations in which the contrast between areas was either decreased (thus approaching a true silhouette) or increased (the light areas became lighter, and the dark areas became darker).

\section{EXPERIMENT 1}

\section{Method}

Subjects. The subjects were 4 feral pigeons that were maintained at $85 \%$ of their free-feeding weights by controlled daily feeding. Prior to the onset of the present experiment, the pigeons had participated in unrelated studies.

Apparatus. The pigeons were trained in four specially constructed plywood chambers. One side of each chamber consisted of a large opening with an aluminum frame attached to the outside of the box. Inside the frame was a clear touch screen (Accutouch Model 002744-FTM-K1, Elographics, Oak Ridge, TN) that was coated with mylar for durability. The subjects' pecks to the touch screen were processed by a serial controller board (Model E271-2210, Elographics). A brushed aluminum panel was placed directly in front of the screen to allow subjects access to limited portions of the video monitor. There were five openings in the aluminum panel. The center opening was a $7 \times 7 \mathrm{~cm}$ square opening in which the object stimuli appeared. The remaining four openings were circular, $1.9 \mathrm{~cm}$ in diameter, and located $2.3 \mathrm{~cm}$ from each corner of the center display opening. The four corner keys served as report keys. In the rear of the chamber, a clear Plexiglas food container was placed level with a wire-mesh floor to prevent subjects from perching on the food cup. Noyes 45-mg pigeon pellets were delivered through a vinyl tube into the food cup, using a rotary pellet dispenser (Model ENV-203M, MED Associates, Lafayette, IN). During experimental sessions, constant illumination was provided by a houselight mounted on the upper rear wall of the chamber. A digital I/O interface board (National Instruments Model NB-DIO-24, Austin, TX) controlled the pellet dispenser and the houselight.

Control of peripheral stimuli (via the I/O interface) and recording of the pigeons' responses (via the serial controller board) were accomplished by four Apple Macintosh 7100/66 Power PC computers equipped with 15 -in. monitors set at $640 \times 480$ resolution. The pigeon's monitor and an identical monitor located in an adjacent room were connected by a distribution amplifier (Model MAC/2 DA2, Extron Electronic, Sante Fe Springs, CA). The programs were developed in Hypercard 2.3.

Stimuli. The stimuli consisted of an arch, a barrel, a brick, and a wedge that varied from one another by a variety of nonaccidental properties (Biederman, 1987). The training stimuli ranged in size from 2 to $4 \mathrm{~cm}$ in width and from 2 to $4 \mathrm{~cm}$ in height; the area of each geon was approximately equal $\left(4.6,4.3,4.2\right.$, and $4.6 \mathrm{~cm}^{2}$ for the arch, barrel, brick, and wedge, respectively). The average grayscale for each geon was also similar $(181,169,184$, and 145 for the arch, barrel, brick, and wedge, respectively; gray-scale saturation could range from 0 for white, to 255 for black). The stimuli were rendered in Raydream Designer 4, using the 300-dpi setting.

The test stimuli consisted of line drawings, silhouettes, and lightchange stimuli that were created in Adobe Photoshop 4.0 (Fig- ure 1). The line drawings were rendered by tracing the edges of each stimulus. The silhouettes were rendered by replacing the internal structure of each stimulus with the median image gray-scale level (175). The light-change stimuli were rendered by rotating the light source approximately $90^{\circ}$ relative to the center of each stimulus (this was done by using Raydream Designer); changing the light position changed the shading of the stimuli while having a minimal effect on gray-scale level for each geon (the average change in grayscale level was a decrease in 6 on the 0 to 255 scale).

Procedure. At the beginning of a trial, the center display area was illuminated with a black cross centered on a white background. A single peck anywhere within that display area turned on a singlegeon stimulus in the center. The pigeons were required to peck the center screen a fixed number of times (observing responses); the fixed ratio varied for different birds, depending on what level they would tolerate, and ranged from 15 to 30 pecks per trial. After the final peck, the four corner report keys were illuminated; each key changed from black to red, green, blue, or yellow, depending on the specific report key (the location of the color was consistent across computers). After a correct choice, the stimulus was removed from the display area, the report keys were darkened, and a food pellet reinforcer was delivered. After an incorrect choice, the stimulus flashed off for $1 \mathrm{sec}$, the report keys were darkened, the houselight was turned off for $4-6 \sec (M=5 \mathrm{sec})$, during which the stimulus was present, and one or more correction trials began (repeating the incorrect trial until a correct choice was made), which were not scored for analysis. After a correct response was produced (whether that response was the first response or followed a number of incorrect responses), there was an intertrial interval ranging from 6 to $10 \mathrm{sec}(M=8 \mathrm{sec})$, during which the houselight remained on and the display was dark.

The experiment consisted of two phases: training and testing. During training, the pigeons were trained to peck one of the four corner report keys for each of the four single-geon objects (see the left column of Figure 1). The assignment of correct report key assignments to the four different geons was counterbalanced using a Latin-square design. Assignment of a geon to a particular report key was constant throughout training.

In training, we used a randomized block design. Each block consisted of five presentations of the four objects; thus, there was a total of 20 trials in a single block. There were 10 blocks in each daily session, for a total of 200 trials per day. Each bird was required to meet a session criterion of $85 \%$ correct overall and $80 \%$ correct for each of the four geons before it could proceed to testing.

During the testing phase, trials on which test stimuli were given were nondifferentially reinforced. That is, the pigeons were given reinforcement for any choice on testing trials, so that repeated testing with the same stimuli would not train the birds to make the correct response (if they were differentially reinforced) or cause the birds to extinguish responding to the testing stimuli (if they were not reinforced). If a pigeon failed to meet the $85 / 80$ criterion for the training trials by the end of a session, it was returned to training for later sessions until it again met the $85 / 80$ criterion. This progression continued until each pigeon had received 20 testing sessions.

In testing, the pigeons saw the line drawings, silhouettes, and light-change stimuli that were created using the four trained stimuli as the templates. This process yielded a total of 12 testing stimuli that were each shown twice within a single session.

Testing sessions began with 40 warm-up trials (4 different stimuli, each shown 10 times). The remainder of the testing session followed a randomized block design. Each block consisted of 23 trials, with 20 of the trials involving the original training views (4 different stimuli each shown 5 times) and the remaining 3 trials involving the testing stimuli. The 3 testing stimuli were chosen randomly without replacement from the total pool of 12 testing stimuli (3 testing versions of four different geons). After all 12 of the testing stimuli had been presented once, the pool was reset, and se- 


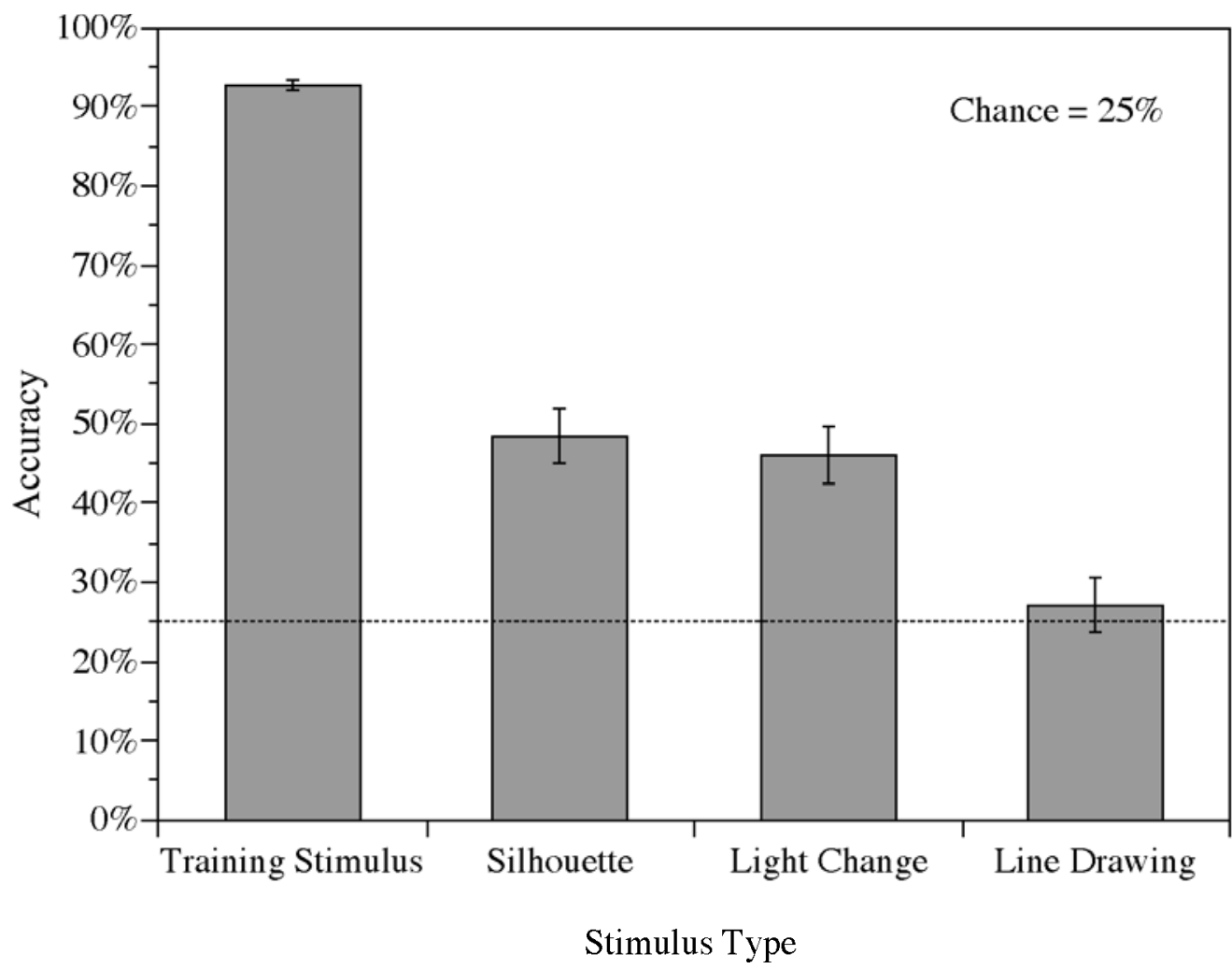

Figure 2. Mean accuracy (with accompanying standard error bars) for each stimulus type during the testing phase of Experiment 1.

lection progressed until each stimulus had been chosen again. There were eight blocks in each daily session, for a total of 224 trials $(40$ differentially reinforced warm-up trials, 160 differentially reinforced training trials, and 24 nondifferentially reinforced testing trials).

\section{Results}

The pigeons took a mean of 29.8 days to complete the training phase. They required a mean of 3.0 additional training sessions during the testing phase (recall that the pigeons were put back on training when they failed to meet the $85 / 80$ criterion).

In testing, the pigeons performed well to the training stimuli $(M=93 \%)$, but they exhibited a large decrement in accuracy to all three types of testing stimuli (Figure 2). For the silhouette and light-change stimuli, accuracy dropped to $48 \%$ and $46 \%$, respectively. Even though this was a large decrement from the training stimuli (a difference of $45 \%$ for the silhouettes and $47 \%$ for the lightchange stimuli), these accuracy scores were still above chance (one-tailed binomial, $p<.001$ ). In contrast, responding to the line drawings was $27 \%$ correct, which was not above chance (one-tailed binomial, $p>.05$ ).

In order to confirm these initial observations, the data from testing were analyzed using a repeated measures analysis of variance (ANOVA) of stimulus type (training, line drawing, silhouette, or light change). The pi- geons showed a significant main effect for stimulus type $[F(3,18)=50.85, p<.001]$.

In order to confirm the observed generalization decrement from the training stimuli to the transformed stimuli, we performed a series of planned comparisons. All three types of testing stimuli produced a statistically significant decrease in accuracy, relative to the trained stimuli (silhouettes, $t=5.16, p<.001$; light-change stimuli, $t=5.45$, $p<.001$; and line drawings, $t=7.64, p<.001$ ).

We also examined the pigeons' mistakes for both the training and the transformed stimuli, but this analysis revealed only that the pigeons had a very strong tendency to adopt a "default" response under conditions of uncertainty (i.e., to choose the same key-e.g., the lower left-for the overwhelming majority of trials in which a misclassification was made).

\section{Discussion}

Pigeons that were trained with realistically illuminated, gray-scale, two-dimensional renderings of simple three-dimensional volumes (geons) exhibited significant transfer to silhouettes of those objects and to versions in which the direction of the light source had been changed by approximately $90^{\circ}$, with no apparent difference in choice accuracy between the silhouette and the lightchange stimuli. Although we observed significant transfer 
No Contrast
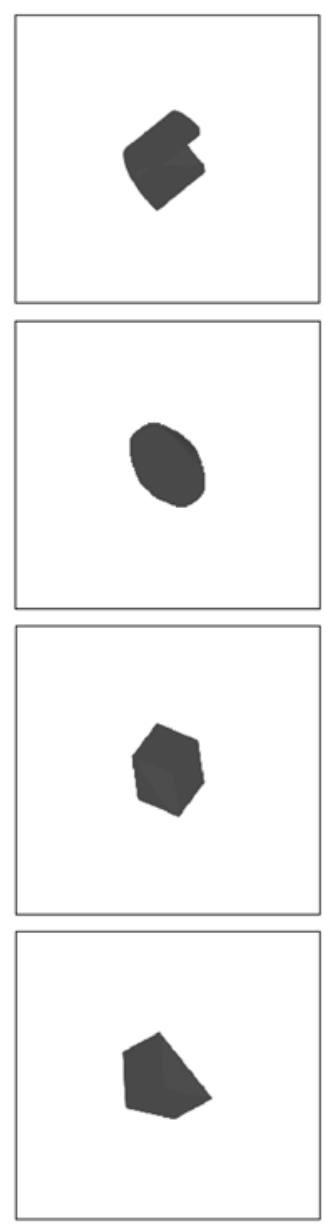

Contrast -15
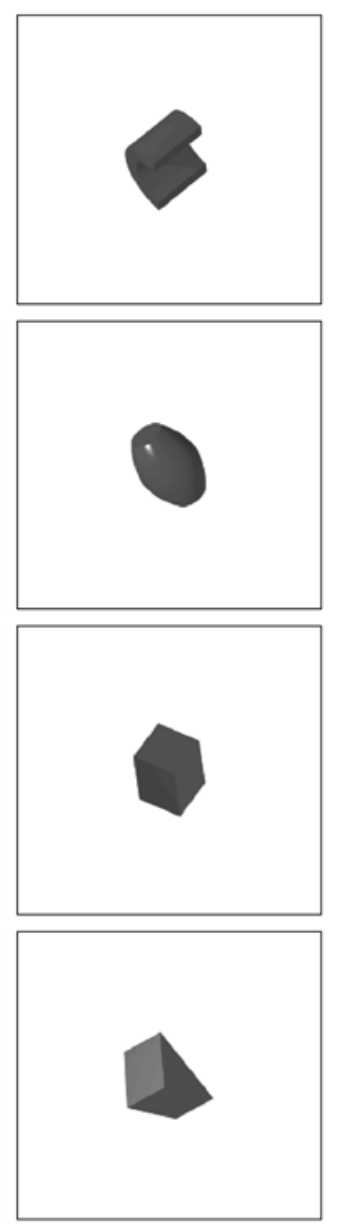

Original
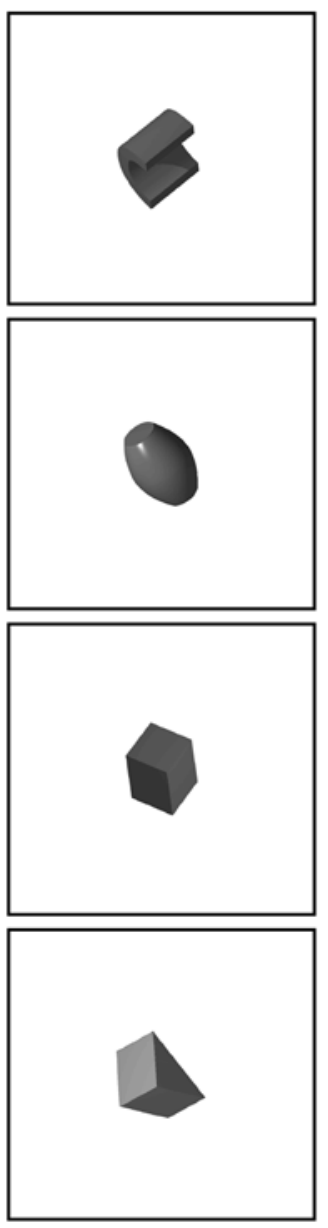

Contrast +25
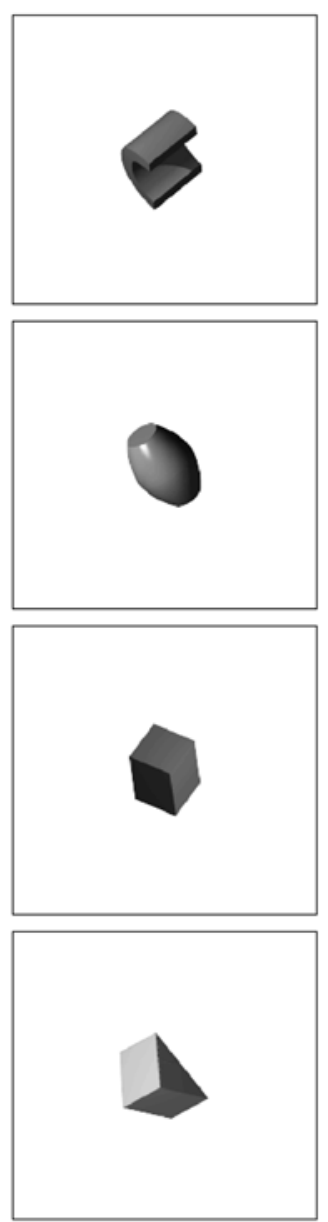

Contrast +50
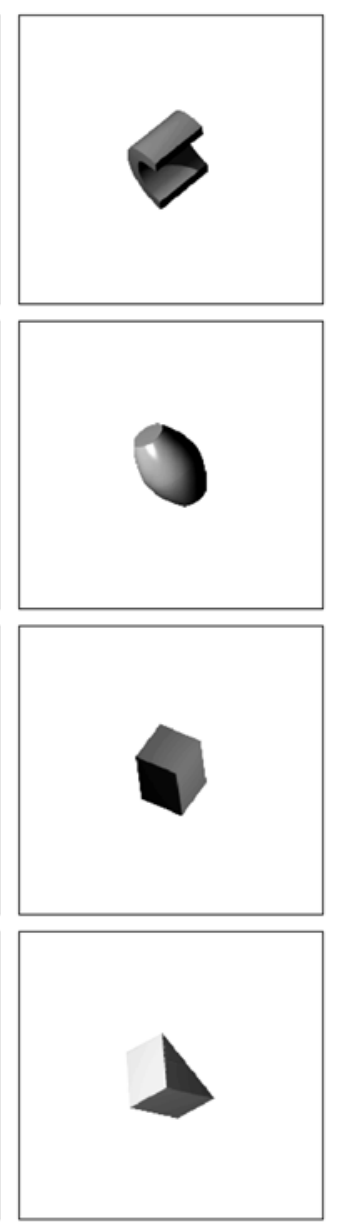

Figure 3. The original and transformed versions of the $0^{\circ}$ rotations of the arch, barrel, brick, and wedge geons used in Experiment 2. The original views (middle column) are those used in training.

(as revealed by above-chance choice accuracy), the pigeons did show a significant decrement in their choice accuracy for these transformed stimuli. In contrast, the pigeons exhibited chance discrimination performance for line drawing equivalents of the objects.

These results indicated that the learned representations of these objects appear to be highly stimulus specific. Even changes that preserved the internal structure of the object and involved only changes in the shading of the object's faces (the light changes) produced a significant decrement in performance. This effect was especially remarkable because personal observation of the ongoing experiment revealed that the experimenters did not recognize which stimuli involved a light change and which ones did not; it was only through very careful observation or with side-by-side comparison that the light change was apparent.

Given the suggested specificity of the learned representation that was revealed in Experiment 1, we wished to determine whether there existed transformations in- volving large changes in the stimulus that nevertheless maintained good discrimination. Because the light-change stimuli were expected to have produced the greatest generalization, we focused on a possible explanation of the observed generalization decrement: These stimuli involved a change in the relative shading of an object's areas. For example, some of the faces of the brick became darker and others became lighter after moving the light source (see Figure 1). Therefore, in Experiment 2, we tested a new group of pigeons with stimulus transformations that preserved the relative shading of the object's areas but that still involved large changes in the stimulus (see Figure 3).

\section{EXPERIMENT 2}

\section{Method}

Subjects. The subjects were 3 feral pigeons that were different from those used in Experiment 1. The pigeons were maintained at $85 \%$ of their free-feeding weights by controlled daily feeding. Prior 
Table 1

The Mean Difference Scores (Measured in Terms of the Mean Absolute Difference in Pixel Gray Levels) for Each of the Transformations Relative to the Original Training Stimuli

\begin{tabular}{lc}
\hline Transformation & Mean Difference Score \\
\hline Line drawing & 15.7 \\
Silhouette & 2.9 \\
Light-change & 3.8 \\
No contrast & 3.0 \\
-15 & 1.4 \\
+25 & 2.4 \\
+50 & 4.6 \\
\hline
\end{tabular}

Note-As a baseline of comparison, the difference scores for a geon compared with itself is 0.0 , and those between entirely different geons ranged from 6.0 to 10.7 .

to the onset of the present experiment, the pigeons had been trained on the four-geon discrimination and tested on size transformations of the stimuli (Peissig et al., 1999b).

Apparatus. The apparatus was identical to that used in Experiment 1.

Stimuli. The stimuli again consisted of an arch, a barrel, a brick, and a wedge. The training stimuli were slightly smaller than those used in Experiment 1 (75\% as large in height and width, 56\% as large in area). The stimuli ranged in size from 1.5 to $3.0 \mathrm{~cm}$ in width and from 1.5 to $3.0 \mathrm{~cm}$ in height. The area of each geon was again approximately equal $\left(2.6,2.4,2.4\right.$, and $2.6 \mathrm{~cm}^{2}$ for the arch, barrel, brick, and wedge, respectively); the stimuli were smaller than those used in Experiment 1 because the new stimuli were used for the earlier testing involving size invariance. The average gray-scale for each geon was identical to that used in Experiment 1.

The test stimuli consisted of increases and decreases of contrast between the light and the dark areas of the stimuli (see Figure 3). The original stimuli were transformed in Adobe Photoshop 4.0, using the curve-fitting tool. ${ }^{2}$ The stimuli involved either an increase in contrast $(+50$ or +25$)$ or a decrease in contrast $(-15$ or no contrast). The no-contrast stimuli were included to replicate Experiment 1's silhouette test and as a baseline for comparing the results of Experiment 2 with those of Experiment 1.

In order to ensure that the contrast transformations of Experiment 2 involved at least some transformations that produced a large change in the image (relative to the changes that were produced in Experiment 1), we derived raw difference scores for the transformations that were used in each experiment. Raw difference scores were determined by comparing the absolute difference in pixel gray level at each pixel location for pairs of images (the original and a transformed image) and averaging these absolute differences; the images included the background (a $7 \times 7 \mathrm{~cm}$ image) so that geons with different shapes could be compared. The image comparison was accomplished by first using the absolute difference function of the NIH Image program that is available at http://rsb.info.nih.gov/ nih-image and then averaging the gray levels of the resulting difference image. The results are shown in Table 1. Although most of the contrast transformations produced a smaller mean difference score than those used in Experiment 1, the +50 contrast stimuli produced a larger mean difference score than did all but the line drawings. Thus, if the pigeons were relying on rudimentary template matching to solve the discrimination, performance on the +50 contrast trials should be worse than the silhouette and light-change stimuli of Experiment 1 and the no-contrast stimuli of Experiment 2, whereas performance on the trials involving the other contrast levels should be better.

Procedure. Individual trials proceeded identically to those in Experiment 1. The experiment consisted of two phases: training and testing. The pigeons were trained to peck one of the four corner report keys for each of the four single-geon objects (see the middle column of Figure 3). The assignment of correct report key assignments to the four different geons was partially counterbalanced across the 3 birds, using a Latin-square design.

Immediately following the earlier size invariance testing, the pigeons were returned to training. In training, we used a randomized block design. Each block consisted of five presentations of a single view of the four objects; thus, there were a total of 20 trials in a single block. There were eight blocks in each daily session, for a total of 160 trials per day. Each bird was required to meet a session criterion of $85 \%$ correct overall and $80 \%$ correct for each of the four geons before it could proceed to testing.

During the testing phase, if a pigeon failed to meet the $85 / 80$ criterion for the training trials by the end of a session, it was returned to training for later sessions until it again met the $85 / 80$ criterion. This progression continued until each pigeon had received 20 testing sessions.

Testing sessions began with 20 warm-up trials (4 different stimuli, each shown five times). The remainder of the testing session followed a randomized block design. Each block consisted of 28 trials, with 24 of the trials involving the original training views (4 different stimuli, each shown six times) and the remaining 4 trials involving the testing stimuli. The 4 testing stimuli were chosen randomly without replacement from the total pool of 20 testing stimuli (4 testing versions of four different geons and the 4 original training stimuli). The original training stimuli were given as test stimuli in order to provide equivalent numbers of testing trials with all the stimuli for comparison. There were five blocks in each daily session, for a total of 160 trials (20 differentially reinforced warmup trials, 120 differentially reinforced training trials, and 20 nondifferentially reinforced testing trials).

\section{Results}

The 3 pigeons in Experiment 2 took a mean of 25.0 days to complete the training phase. The pigeons required a mean of 5.0 additional training sessions during the testing phase (recall that the pigeons were put back on training when they failed to meet the $85 / 80$ criterion).

In testing, the pigeons performed well on the nondifferentially reinforced trials with the training stimuli $(M=$ $92 \%$ ) and to many of the contrast-changed stimuli (Figure 4). They exhibited a small numerical drop in accuracy to the stimuli with less severe changes in contrast $(+25$ contrast and -15 contrast), and a larger drop in accuracy to the stimuli with more severe changes in contrast $(+50$ contrast and no contrast stimuli). For the stimuli in which the contrast was increased, the pigeons' accuracy fell to $90 \%$ for +25 contrast stimuli and to $75 \%$ for +50 contrast stimuli. For the stimuli in which the contrast was decreased, the pigeons' accuracy fell to $83 \%$ for -15 contrast stimuli and to $40 \%$ for no contrast stimuli. The pigeons performed significantly above chance for all of the test stimuli (one-tailed binomial, $p$ s $<.001$ ).

The data from testing were analyzed using a repeated measures, full factorial ANOVA of stimulus type (training, +50 contrast, +25 contrast, -15 contrast, and no contrast). The pigeons showed a significant main effect for stimulus type $[F(4,8)=16.73, p<.001]$.

In order to confirm the observed pattern of generalization from the training stimuli to the transformed stimuli, we performed a series of planned comparisons. The pigeons exhibited a statistically significant decrease in accuracy from the trained stimuli to the +50 contrast and the 


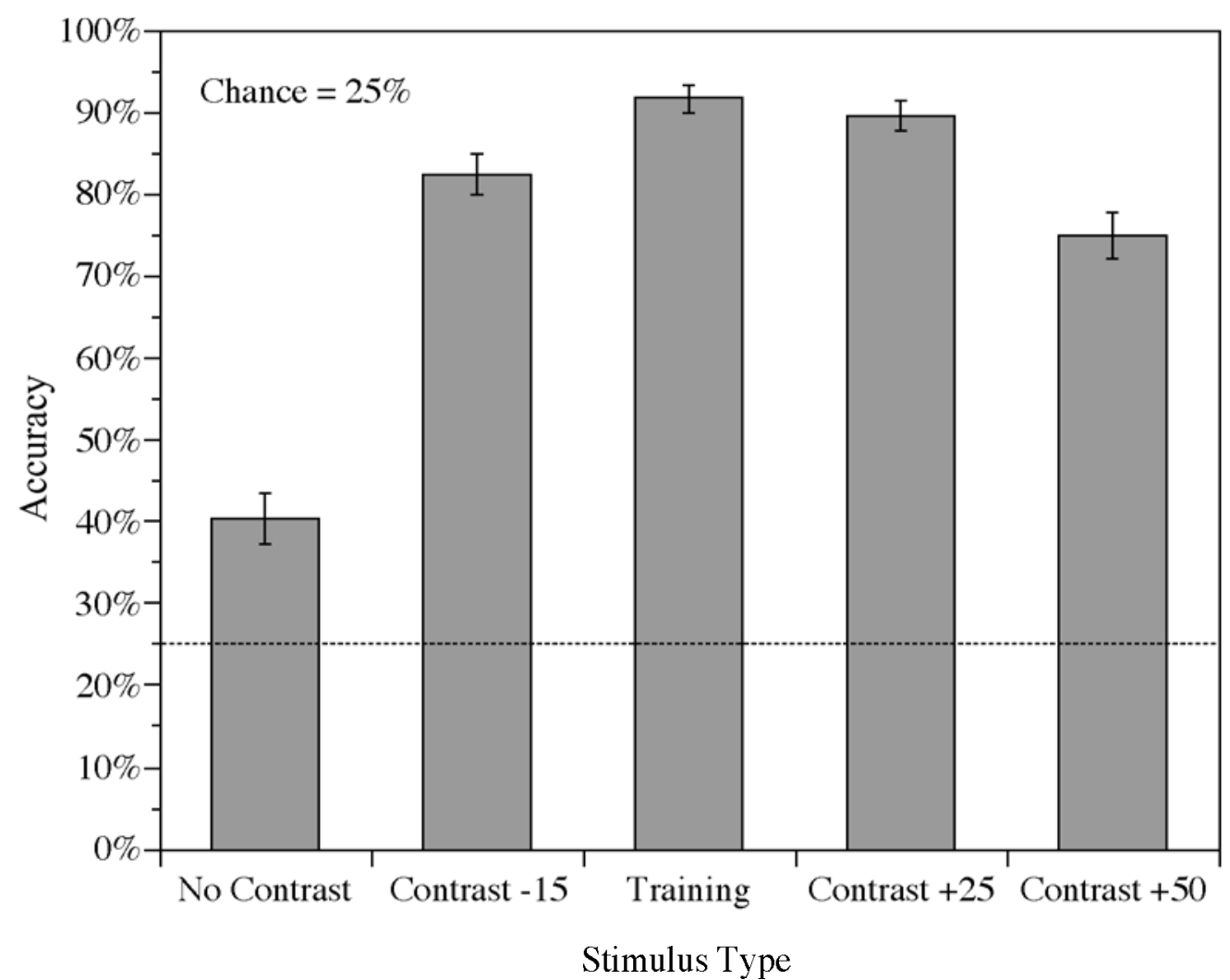

Figure 4. Mean accuracy (with accompanying standard error bars) for each stimulus type during the testing phase of Experiment 2.

no-contrast test stimuli ( +50 contrast stimuli, $t=2.33$, $p<.05$; no-contrast stimuli, $t=7.11, p<.001)$. However, the pigeons' response accuracies to the +25 contrast and -15 contrast test stimuli were not significantly different from that to the trained stimuli $(+25$ contrast stimuli, $t=0.30, p>.05 ;-15$ contrast stimuli, $t=1.27, p>$ $.05)$. Importantly, response accuracy to the +50 contrast stimuli was significantly better than that to the no-contrast stimuli $(t=4.78, p<.01)$, thus revealing that a larger absolute difference score ( 4.6 for +50 contrast stimuli; see Table 1) produced better generalization than a smaller absolute difference score (3.0 for no contrast stimuli).

\section{Discussion}

The results of Experiment 2 clearly indicate that a change that looks quite pronounced to the human eye and is quite large in terms of absolute pixel differences $(+50$ contrast stimuli) can nonetheless support strong generalization of discriminative performance in the pigeon. This finding contrasts with that involving the light-change stimuli of Experiment 1, in which the changes seemed to be quite minimal to the human eye and in terms of absolute pixel differences but still produced a large generalization decrement in the pigeon.

The combined results of these two experiments suggest that the relative brightness of each surface contour, as compared with the other contours of the object, is more important for recognition than is the absolute brightness of each of these contours. For example, the darkest face of the brick geon that the pigeons saw during training (the lower left face, first column of Figure 1) is no longer the darkest face after a light change (where the darkest face is now the lower right face, second column of Figure 1); however, the lower left face is still the darkest face after any of the contrast transformations (second through fifth columns of Figure 3) except for no contrast. The absolute brightness of each contour (e.g., each face of the brick) is altered by both the light-change and the contrast transformations; however, the contrast transformation maintains the relative ordering of contour brightness. This account suggests that the removal of all contrast produces much poorer recognition performance (discrimination accuracy of $48 \%$ in Experiment 1 and $40 \%$ in Experiment 2) because all information about the relative brightness of surface contours is lost - the birds must thereby rely solely on the outline shape of the object.

\section{GENERAL DISCUSSION}

Pigeons that were trained with realistically illuminated, gray-scale, two-dimensional renderings of simple three-dimensional volumes (geons) exhibited significant 
transfer to silhouettes of those objects and to versions in which the direction of the light source had been changed by approximately $90^{\circ}$ (Experiment 1 ). The pigeons also showed very strong transfer to transformations that increased or decreased the lighting contrast, as long as some degree of contrast was still present (Experiment 2). Although we observed significant transfer (as revealed by above-chance choice accuracy), the pigeons did show a significant decrement in their choice accuracy to many of these transformed stimuli. In contrast, the pigeons exhibited chance discrimination performance to the line drawing equivalents of the objects (Experiment 1).

Contrary to previous studies involving human subjects, our experiment revealed that pigeons evidence an acute sensitivity to the internal surface features of a twodimensional representation of an object. The most surprising result in our study was the large decrement in accuracy that was caused by changes in the direction of lighting (in our personal observations of the program as it was presenting the stimuli, we usually failed to notice that the light-changed stimuli were not the original training stimuli). In another study exemplifying sensitivity to internal surface features, Reid and Spetch (1998) tested the pigeon's ability to use surface features to discriminate which of two two-dimensional images was a valid representation of a three-dimensional object. They found that pigeons can use internal surface variations in a way that is consistent with the classification of images as valid representations of three-dimensional objects, thus evidencing sensitivity to the configuration of these internal surface features.

The present work highlights both the specificity and the generality of the pigeon's learning in a discrimination task involving two-dimensional representations of threedimensional objects. In our task, the pigeons readily encoded details of the internal structure of the images and showed significant decrements when certain aspects of this structure were changed (Experiment 1). The pigeons did, however, show some flexibility in their derived stimulus categories, as is evidenced by the relatively small effects of substantial changes in contrast (Experiment 2).

The present research contributes to an ongoing enterprise intended to determine which features of a twodimensional representation are being used by the pigeon in these discrimination tasks (see Fetterman, 1996, for a discussion of the importance of defining the effective stimulus). We now know that relevant features are well maintained when a stimulus increases or decreases in size up to a factor of about 2.0 (Cerella, 1990; Lombardi \& Delius, 1990; Peissig et al., 1999b) when an object is rotated in depth up to about $72^{\circ}$ (although this result is highly geon specific; Peissig et al., 1999a, 2000; Wasserman et al., 1996), when a stimulus is rotated to any degree in the plane (Delius \& Hollard, 1995; Hollard \& Delius, 1982), and when the contrast is changed (Experiment 2 of the present study). In contrast, large depth rotations and size changes produce correspondingly large decrements in discriminative performance, as does the removal of all contrast, a shift in lighting, and the removal of texture (thus producing a line drawing).

Collectively, results involving realistically illuminated, gray-scale, two-dimensional renderings of threedimensional volumes suggest that the pigeon is primarily attending to global shading properties of these twodimensional stimuli; these properties include both the relative brightness and the location of the shading contours that make up an object. Rotating an object in depth, moving the light source, removing contrast, and substantially changing the object's size will have profound effects on the relative brightness and location of these contours, whereas changing the contrast level will not. Note that planar rotations might also be expected to have a large effect on recognition of our simple geons because the locations of the shading contours will change under rotation. Although some previous work has demonstrated that planar rotations produce relatively small decrements in discriminative performance, the stimuli used in these studies have involved geometric shapes with uniform color (Delius \& Hollard, 1995; Hollard \& Delius, 1982) or complex stimuli involving myriad parts (e.g., human faces; Jitsumori \& Yoshihara, 1997). Consistent with our interpretation, however, research under way in our laboratory is revealing that planar rotation of our realistically rendered geons produces large decrements in recognition accuracy.

Although we believe that our pigeons are using relative brightness of surface contours as an important discriminative cue, our data also clearly indicate that the outline shape of an object is likewise encoded, as is evident in the above-chance performance on the silhouettes in both experiments. Indeed, the pigeon is clearly able to discriminate visual stimuli on the basis of outline shape alone (Delius \& Hollard, 1995; Hollard \& Delius, 1982), as well as stimuli involving no shading whatsoever (e.g., with line drawings; Cerella, 1977; Delius \& Hollard, 1995; Wasserman et al., 1996; Wasserman et al., 1993). This earlier research, as well as work currently under way in our laboratory, suggests that pigeons are flexible recognizers; the effective discriminative cues in a visual recognition task substantially differ, depending on the nature of the stimuli to be discriminated.

Previous and current investigations demonstrate that any theory of avian object recognition must account for both the pigeon's ability to place similar objects into the same category and its differential sensitivity to changes in the myriad features of those objects. These findings also suggest that pigeons see the world somewhat differently than we do. Any theory of object recognition that is derived from studies involving humans (e.g., recognition-bycomponents; Biederman, 1987) will require a change in the relative effects of image features before it can be successfully applied as a complete model of the pigeon's recognition of objects. 


\section{REFERENCES}

Bhatt, R. S., \& WASSERman, E. A. (1989). Secondary generalization and categorization in pigeons. Journal of the Experimental Analysis of Behavior, 52, 213-224.

Biederman, I. (1987). Recognition-by-components: A theory of human image understanding. Psychological Review, 94, 115-147.

Biederman, I. (1988). Aspects and extensions of a theory of human image understanding. In Z. Pylyshyn(Ed.), Computationalprocesses in human vision (pp. 370-428). New York: Ablex.

Biederman, I., \& BAR, M. (1999). One-shot viewpoint invariance in matching novel objects. Vision Research, 39, 2885-2899.

Biederman, I., \& Ju, G. (1988). Surface versus edge-base determinants of visual recognition. Cognitive Psychology, 20, 38-64.

CABE, P. A. (1976). Transfer of discrimination from solid objects to pictures by pigeons: A test of theoretical models of pictorial perception. Perception \& Psychophysics, 19, 545-550.

Cerella, J. (1977). Absence of perspective processing in the pigeon. Pattern Recognition, 9, 66-68.

Cerella, J. (1990). Shape constancy in the pigeon: The perspective transformations decomposed. In M. L. Commons et al. (Eds.), Behavioral approaches to pattern recognition and concept formation (Quantitative Analyses of Behavior, Vol. 8, pp. 145-163). Hillsdale, NJ: Erlbaum.

CooK, R. G., \& Katz, J. S. (1999). Dynamic object perception by pigeons. Journal of Experimental Psychology: Animal Behavior Processes, 25, 194-210.

Cook, R. G., Wright, A. A., \& Kendrick, D. F. (1990). Visual categorization by pigeons. In M. L. Commons et al. (Eds.), Behavioral approaches to pattern recognition and concept formation (Quantitative Analyses of Behavior, Vol. 8, pp. 187-214). Hillsdale, NJ: Erlbaum.

Delius, J. D. (1992). Categorical discrimination of objects and pictures by pigeons. Animal Learning \& Behavior, 20, 301-311.

Delius, J. D., \& Hollard, V. D. (1995). Orientation invariant pattern recognition by pigeons (Columba livia) and humans (Homo sapiens). Journal of Comparative Psychology, 109, 278-290.

Fetterman, J. G. (1996). Dimensions of stimulus complexity. Journal of Experimental Psychology: Animal Behavior Processes, 22, 3-18.

HaYWARD, W. G. (1998). Effects of outline shape in object recognition. Journal of Experimental Psychology: Human Perception \& Performance, 24, 427-440.

Hollard, V. D., \& Delius, J. D. (1982). Rotational invariance in visual pattern recognition by pigeons and humans. Science, 218, 804-806.

JiTSUMORI, M., \& YoshiHARA, M. (1997). Categorical discrimination of human facial expressions by pigeons: A test of the linear feature model. Quarterly Journal of Experimental Psychology, 50B, 253268.

Kirkpatrick-Steger, K., \& Wasserman, E. A. (1996). The what and where of the pigeon's processing of complex visual stimuli. Journal of Experimental Psychology: Animal Behavior Processes, 22, 60-67.

Lombardi, C. M., \& Delius, J. D. (1988). Pattern recognition invariance in pigeons (Columba livia). International Journal of Comparative Psychology, 2, 83-102.

Lombardi, C. M., \& Delius, J. D. (1990). Size invariance in visual pattern recognition by pigeons. In M. L. Commons et al. (Eds.), Behavioral approaches to pattern recognition and concept formation (Quantitative Analyses of Behavior, Vol. 8, pp.41-65). Hillsdale, NJ: Erlbaum.

LUMSDEN, E. A. (1977). Generalization of an operant response to photographs and drawings/silhouettes of a three-dimensional object at various orientations. Bulletin of the Psychonomic Society, 10, 405407.

Newell, F. N., \& Findlay, J. M. (1997). The effect of depth rotation on object identification. Perception, 26, 1231-1257.

Peissig, J. J., Young, M. E., Wasserman, E., \& Biederman, I. (1999a).
The pigeon's perception of depth-rotated shapes. Cahiers de Psychologie Cognitive, 18, 657-690.

Peissig, J. J., Young, M. E., Wasserman, E. A., \& Biederman, I. (1999b, November). Stimulus size and the pigeon's ability to recognize objects. Poster presented at the 40th Annual Meeting of the Psychonomic Society, Los Angeles.

Peissig, J. J., Young, M. E., Wasserman, E. A., \& Biederman, I. (2000). Seeing things from a different angle: The pigeon's recognition of single geons rotated in depth. Journal of Experimental Psychology: Animal Behavior Processes, 26, 115-132.

Reid, S. L., \& Spetch, M. L. (1998). Perception of pictorial depth cues by pigeons. Psychonomic Bulletin \& Review, 5, 698-704.

Sekuler, A. B., Lee, J. A. J., \& Shettleworth, S. J. (1996). Pigeons do not complete partly occluded figures. Perception, 25, 1109-1120.

TARr, M. J., Bülthoff, H. H., Zabinski, M., \& Blanz, V. (1997). To what extent do unique parts influence recognition across changes in viewpoint? American Psychological Society, 8, 282-288.

TARr, M. J., Kersten, D., \& Bülthoff, H. H. (1998). Why the visual recognition system might encode the effects of illumination. Vision Research, 38, 2259-2275.

Wasserman, E. A., Gagliardi, J. L., Cook, B. R., Astley, S. L., Kirkpatrick-Steger, K., \& Biederman, I. (1996). The pigeon's recognition of drawings of depth-rotated stimuli. Journal of Experimental Psychology: Animal Behavior Processes, 22, 205-221.

Wasserman, E. A., Kirkpatrick-Steger, K., Van Hamme, L. J., \& Biederman, I. (1993). Pigeons are sensitive to the spatial organization of complex visual stimuli. Psychological Science, 4, 336-341.

\section{NOTES}

1. Although we use the phrase object recognition to describe both human and pigeon performance on these discriminative tasks, we do not assume that pigeons see these two-dimensional representations as objects in the same way that people do (and our data indicate that they do not). Our stimuli might simply represent two-dimensional patterns from the avian perspective. Regardless, the stimuli are two-dimensional representations of objects; thus, we use the term objects to describe the stimuli that the pigeons are discriminating, without making the stronger claim that these patterns are representing objects in the strictest sense.

2 . Using the curve-fitting tool, we chose a function that would translate gray-scale values (ranging between 0 for black and 255 for white) to new values. Note that Adobe's curve-fitting tool uses saturation scores that are the inverse of those used in our image analysis software (e.g., the average brightness in NIH Image was 175, whereas the average brightness in Adobe PhotoShop was $255-175=80$ ). In all of our contrast transformations, we chose a gray-scale value near the average brightness level of the images (75) as the middle anchor. To increase contrast, values above 75 were increased (making them closer to the upper extreme of 255; the light shades were made lighter), and values below 75 were decreased (making them closer to the lower extreme of 0 ; the dark shades were made darker). To decrease contrast, values above 75 were decreased, and values below 75 were increased (making both closer to the middle value of 75 , thus effectively washing out the extreme values). Two other anchors were chosen at 50 and 100 (representing nearly the entire range of observed gray-scale values of our images). A +25 contrast indicates that the 50 gray-scale value was transformed to a 25 ( 25 points closer to the lower extreme of 0$)$, a 100 gray-scale value was transformed to a 125 ( 25 points closer to the upper extreme of 255), and values between 50 and 100 were scaled as a linear function of their distance from the middle anchor of 75 . Thus, values close to 75 were changed the least, whereas those farthest from 75 were changed the most. Similar transformations were done for the other contrast levels. No-contrast stimuli had a uniform gray-scale value of 75 .

(Manuscript received September 5, 2000; revision accepted for publication February 22, 2001.) 